\title{
Hydraulic Losses of Flow Control Devices in Pipes*
}

\author{
Tamotsu IGARASHI** and Susumu INAGAKI**
}

\begin{abstract}
Concerning the vortex shedder used for the Karman vortex flowmeter, the flow resistances of cylindrical bodies of various cross-sections in a circular pipe are given by the general equation in the previous works of Igarashi et al. In the present paper, it is confirmed that the equation is applicable to the vortex shedder having a low opening ratio. Then, the same idea is applied to the hydraulic losses of flow control devices asymmetrical to the cross-section of the pipe, such as a sluice valve, a butterfly valve and a cock. Consequently, these hydraulic losses are expressed by the following general equation: $\zeta=C\left[(1-\beta) / \beta^{2}\right]^{n}$, where $\beta$ is the opening ratio.
\end{abstract}

Key Words: Pipe Flow, Flow Control Devices, Flowmeter, Vortex Shedder, Valve, Hydraulic Loss, Resistance Coefficient, Opening Ratio, Blockage Ratio

\section{Introduction}

In the previous paper ${ }^{(1)}$, the characteristics of the flow resistance of a vortex shedder in a circular pipe are clarified. The vortex shedder used for the Karman vortex flowmeter ${ }^{(2)}$ is a cylindrical body of various shapes and set up in a turbulent flow through the pipe. The flow resistance of the shedder was expressed as a general equation of the function of the opening ratio and the drag coefficient of the shedder in a uniform flow under a wide range of Reynolds numbers.

In the present paper, it is confirmed that the above-mentioned equation applies to a typical vortex shedder, that is, a circular cylinder and a trapezoidal cylinder having low opening ratio. As a result, the vortex shedder can be applied to a very simple and useful flowmeter for measurement of the pressure difference across the vortex shedder instead of the

* Received 21th February, 1994. Japanese original: Trans. Jpn. Soc. Mech. Eng., Vol. 59, No. 561, B (1993), pp. 1480-1485. (Received 7th September, 1992).

** Department of Mechanical Engineering, The National Defense Academy, 1-10-20 Hashirimizu, Yokosuka 239, Japan throttle type flowmeter, for example, an orifice.

Valve and cock are usually set up in a pipe or a duct for the control of flow rate. These have various shapes depending on their applications, and their hydraulic losses are complicated and different each other. The flow resistance coefficients are hitherto given by a chart or a table in Ref. (3). The flow resistance coefficients of various flow control devices in pipes are formulated by the same factor $(1-\beta) / \beta^{2}$ used for the vortex shedders ${ }^{(1)}$.

\section{Nomenclature}

$A$ : frontal area of a vortex shedder in a flow

$A_{0}:$ cross-sectional area in a pipe or duct $=\pi D^{2} / 4$

$C:$ constant

$C_{D}$ : drag coefficient of two-dimensional vortex shedder in a uniform flow

$D:$ inner diameter of a circular pipe

$d$ : characteristic length of a vortex shedder

$g:$ acceleration of gravity

$h$ : hydraulic loss of head $=\zeta\left(U_{m}^{2} / 2 g\right)$

$K$ : coefficient of flow resistance of obstruction $=\Delta p / 0.5 \rho U_{m}^{2}$ 
$L:$ height of sluice valve opening

$\Delta p$ : overall pressure difference

$R e_{c}$ : modified Reynolds number of vortex shedder $=U_{c} d / \nu$

$R e_{d}:$ Reynolds number of vortex shedder $=U_{m} d / \nu$

$R e$ : Reynolds number of pipe flow $=U_{m} D / \nu$

$U_{c}$ : corrected mean velocity around a vortex shedder $=U_{m} / \beta$

$U_{m}:$ mean velocity in a pipe

$\beta$ : opening ratio $=1-A / A_{0}$

$\zeta$ : coefficient of flow resistance of controlling device $=\Delta p / 0.5 \rho U_{m}^{2}$

$\theta:$ angle of valve opening

$\nu, \rho:$ kinematic viscosity and density of fluid

\section{Experimental Apparatus and Procedure}

Experiments were carried out using the same apparatus used in the previous works ${ }^{(1)}$. The straight circular pipe has inner diameter $D=150 \mathrm{~mm}$ and 9590 $\mathrm{mm}$ length and the location of a vortex shedder was $40 D$ downstream from the inner port of the pipe. The differential static pressure was measured $413 \mathrm{~mm}$ upstream and $650 \mathrm{~mm}$ downstream the vortex shedder. The flow in the circular pipe was a fully developed turbulent flow. The vortex shedders used were a circular cylinder and a trapezoidal cylinder as shown in Fig. 1. The projected width of the shedder in the flow direction $d$ ranged from 40 to $90 \mathrm{~mm}$. In the previous paper ${ }^{(1)}$, it ranged from 10 to $40 \mathrm{~mm}$. The opening ratio $\beta$ is defined by the following equation :

$$
\beta=\left(A_{0}-A\right) / A_{0}=1-A / A_{0},
$$

where $A_{0}$ is the cross-sectional area in the pipe and $A$ is the frontal area of the vortex shedder. The opening ratio $\beta$ ranged from 0.660 to 0.236 . In order to investigate the Reynolds number effect on the hydraulic losses, the mean velocity, $U_{m}$, was set to range from 2 to $26 \mathrm{~m} / \mathrm{s}$. Then, the Reynolds number of pipe, $R e_{D}=$ $U_{m} D / \nu$, ranged from $1.9 \times 10^{4}$ to $2.5 \times 10^{5}$ and the modified Reynolds number of the vortex shedder, $R e_{c}$ $=U_{c} d / \nu$, ranged from $1.9 \times 10^{4}$ to $5.0 \times 10^{5}$. The corrected mean velocity around the shedder, $U_{c}$, is given by $U_{m} / \beta$. The flow resistance coefficient, $K$, is defined by

$$
K=\Delta p / 0.5 \rho U_{m}^{2}
$$

The resistances coefficients of flow control devices in pipes, that is, a valve, a cock and an orifice

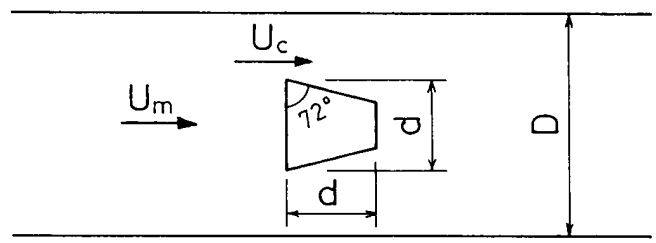

Fig. 1 Flow geometry and cylindrical vortex shedder as shown in Fig. 2, were quoted from Ref.( 3 ) and original papers ${ }^{(5)}$.

\section{Flow Resistance of Cylindrical Bodies}

\section{1 Circular Cylinder and Trapezoidal Cylinder}

A circular cylinder and a trapezoidal cylinder are the most popular shapes of the vortex shedder. The effect of the Reynolds number and the opening ratio $\beta$ on the coefficient of flow resistances (pressure loss coefficients) $K$ is shown in Figs. 3(a) and (b). The value of $K$ increases rapidly with a decrease in the opening ratio. With regard to the Reynolds number effect, up to $R e_{D}=10^{5}$ the value of $K$ of a circular cylinder is nearly constant or slightly decreases with an increase in $R e_{D}$. However a large decrease in $K$ occurs at $R e_{D}>10^{5}$, reaching $70 \%$ of $K$ at $R e_{D} \leqq 10^{5}$. In contrast, the $K$ of a trapezoidal cylinder decreases slightly at $R e_{D}>10^{5}$. Next, we discuss the difference in $K$ of the two vortex shedders when $R e_{D}>10^{5}$. The value of $K$ at $R e_{D}=8.8 \times 10^{4}$ is defined as $K_{0}$. The $R e_{c}$ is defined as $U_{c} d / \nu$, and $U_{c}$ is given by $U_{m} / \beta$. The relationship between $K / K_{0}$ and $\operatorname{Re}_{c}$ is shown in Fig. 4 . The values of $K / K_{0}$ of the trapezoidal cylinder slightly decrease with an increase in $R e_{c}$. On the other hand, the values of $K / K_{0}$ of the circular cylinder decrease rapidly beyond $R e_{c}=2 \times 10^{5}$, which is intimately related with the changes in the flow in the boundary, that is, the transition from laminar to turbulent flow


(a) Sluice valve

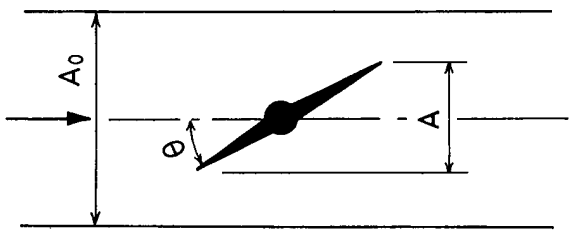

(b) Butterfly valve

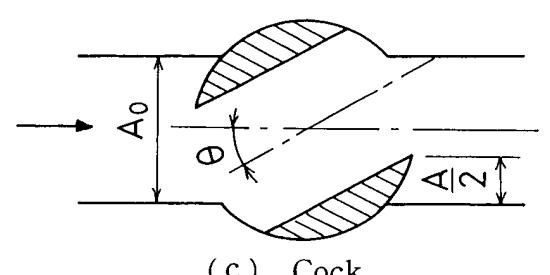

Fig. 2 Pipeline devices 




(a) Circular cylinder

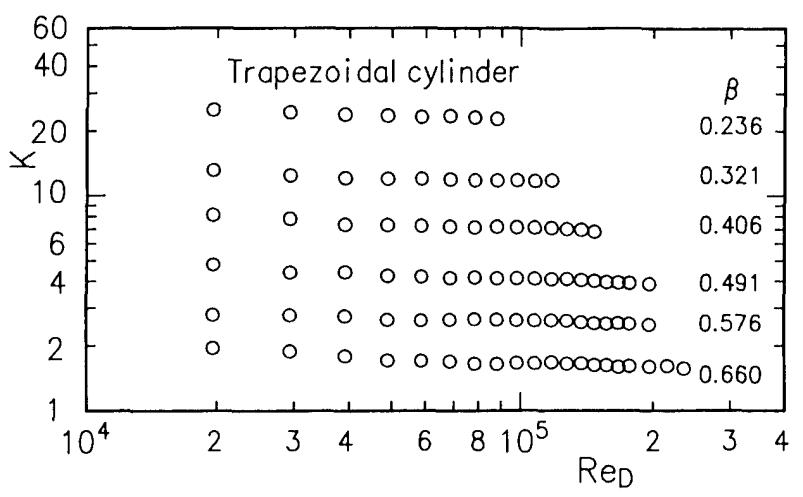

(b) Trapezoidal cylinder

Fig. 3 Effect of Reynolds number and opening ratio on flow resistance coefficient for vortex shedders

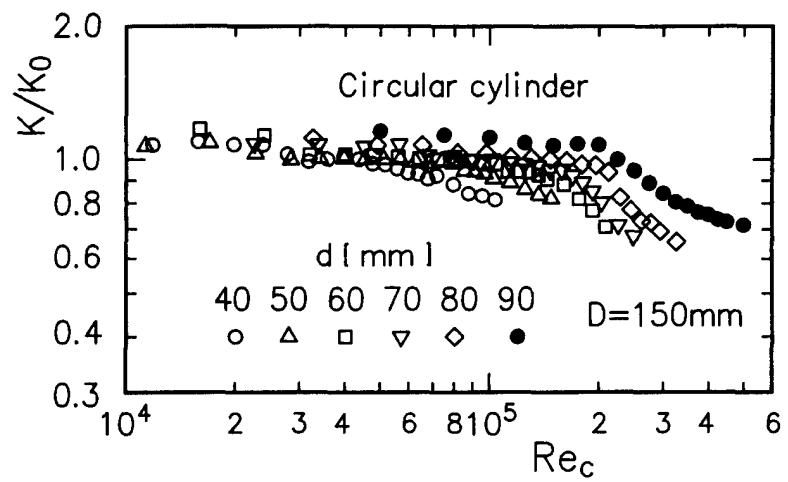

(a) Circular cylinder

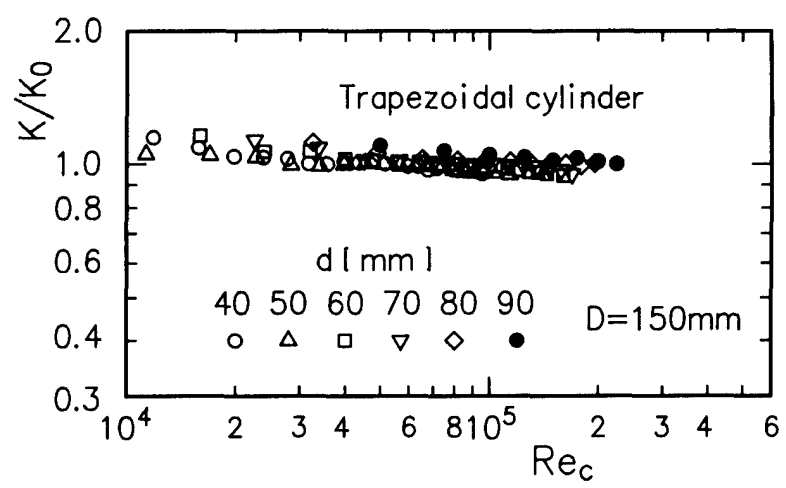

(b) Trapezoidal cylinder

Fig. 4 Effect of modified Reynolds number on flow resistance coefficient for vortex shedders on a circular cylinder in a uniform flow. In fact, from visualization by an oil-film method, the separation point on the circular cylinder is shifted downstream side. However, in the case of the trapezoidal cylinder, the separation always occurs at the front edges of the cylinder. Therefore, the rapid decrease of $K$ of the circular cylinder is caused by the reduction in wake width behind the circular cylinder due to the shift of the separation points.

The results obtained at $R e_{D}=8.8 \times 10^{4}$ and the opening ratio range $\beta=0.236 \sim 0.576$ are shown in Fig. 5 , together with those of the previous paper ${ }^{(1)}$ for $\beta=0.660 \sim 0.915$. The $K$ of a circular cylinder and a trapezoidal cylinder is expressed by

$$
\begin{aligned}
& K=C(1-\beta) / \beta^{2} \\
& C=0.8 C_{D}
\end{aligned}
$$

where $C_{D}$ is the drag coefficient in a uniform flow. Therefore, the value of the constant $C$ is 0.85 for the circular cylinder and 2.0 for the trapezoidal one. The range of the opening ratio in Eq. ( 3 ) is $0.236 \leqq \beta \leqq$ 0.915 , and that of Reynolds number is $1.9 \times 10^{4} \leqq R e_{D} \leqq$ $10^{5}$ for the circular cylinder and $1.9 \times 10^{4} \leqq R e_{D} \leqq 2.0 \times$ $10^{5}$ for the trapezoidal cylinder.

\section{2 Application to a throttle-type flowmeter}

The vortex shedder set up in a pipe can be applied to not only a Karman vortex flowmeter by measuring the vortex shedding frequencies, but also a throttletype flowmeter by measuring the pressure difference. Since, a circular cylinder and a trapezoidal cylinder are simple in construction, they can be applied to a throttle-type flowmeter in a smaller pipe than that of orifice having a minimum diameter of $50 \mathrm{~mm}$. The hydraulic loss defined by following equation

$$
h=\zeta\left(U_{m}^{2} / 2 g\right)
$$

can be transformed into

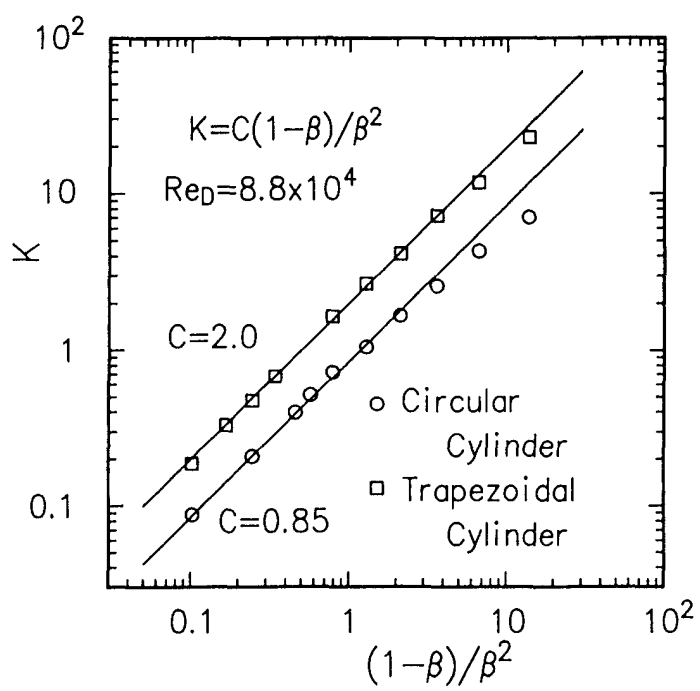

Fig. 5 Flow resistance coefficients for circular cylinder and trapezoidal cylinder 
$\zeta=\rho g h / 0.5 \rho U_{m}^{2}=\Delta p / 0.5 \rho U_{m}^{2}=K$.

Therefore, the hydraulic loss $\zeta$ is equal to $K$. According to $\mathrm{Oki}^{(4)}$, the hydraulic loss of an orifice is given by

$$
\zeta=(1 / \beta-1)(2.75 / \beta-1.56)
$$

The correlation between $(1-\beta) / \beta^{2}$ and $\zeta$ is illustrated in Fig. 6 for an orifice, and compared with that for a trapezoidal cylinder. The hydraulic loss of the trapezoidal cylinder is the same as that of the orifice, while that of a circular cylinder shown in Fig. 5 is below $45 \%$ that of the orifice shown in Fig. 6 . Equation $(7)$ can then be rewritten as

$$
\zeta=1.9\left[(1-\beta) / \beta^{2}\right]^{1.07} \text {. }
$$

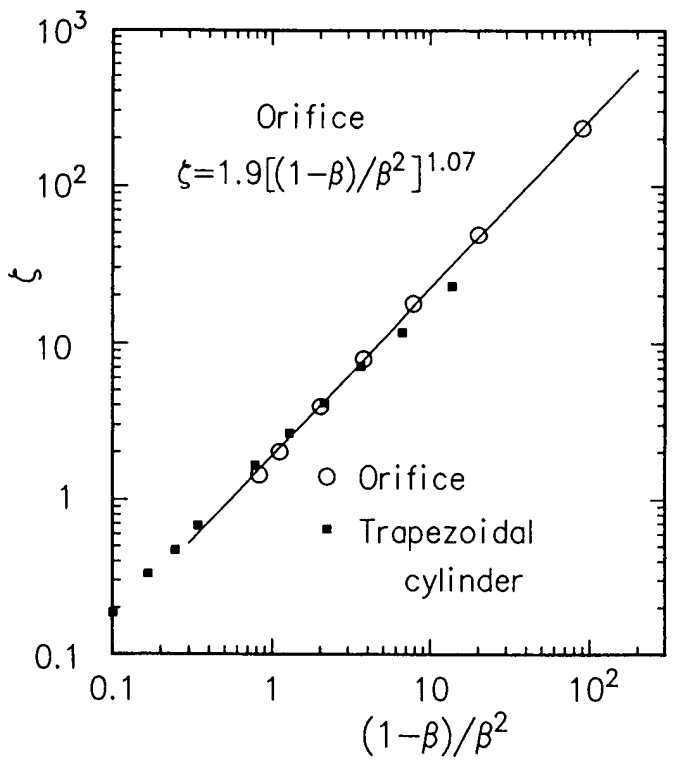

Fig. 6 Comparison between vortex shedders and orifice
It was shown that the factor $(1-\beta) / \beta^{2}$ applies to the hydraulic loss of a three-dimensional body such as an orifice. In the next section, we investigate if the above-mentioned correlation is applicable or not to the various flow control devices in a pipe.

\section{Hydraulic Losses of Flow Control Devices}

\section{1 Resistance coefficients of flow control devices}

The flow control devices in a pipe such as valve and cock are accompanied with flow resistance. In this paper, we deal with the sluice valve, butterfly valve and cock shown in Fig. 2. These devices are asymmetric with respect to the cross-section of the pipe and have either a circular or a rectangular cross ${ }^{-}$ section. The flow resistance coefficients obtained by Weisbach ${ }^{(5)}$ are tabulated in Ref. (3). These data are illustrated by a diagram in Figs. 7 (a) $-(\mathrm{c})$ against the valve opening, $L / d$, or the angle of valve opening, $\theta$. For the sluice valve, the flow resistance coefficients decrease with an increase in $L / d$, and that of rectangular type is larger than that of circular type at the same $L / d$. In the cases of the butterfly valve and cock, the diameter of the circular cross-section is 40 $\mathrm{mm}$ and the rectangular section is $50 \times 25 \mathrm{~mm}$. The flow resistance coefficient of the butterfly valve of circular type is slightly larger than that of rectangular type at the same $\theta$. For the cock, the flow resistance coefficients of rectangular type is slightly larger than that of circular type at the same $\theta$. The difference in the coefficients of the two types increases with an increase in $\theta$.

In the following, we attempt to obtain the

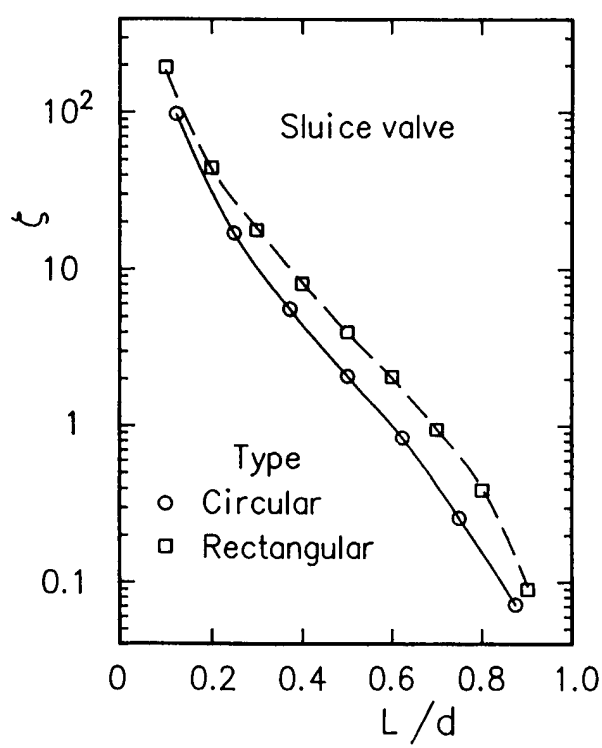

(a) Sluice valve

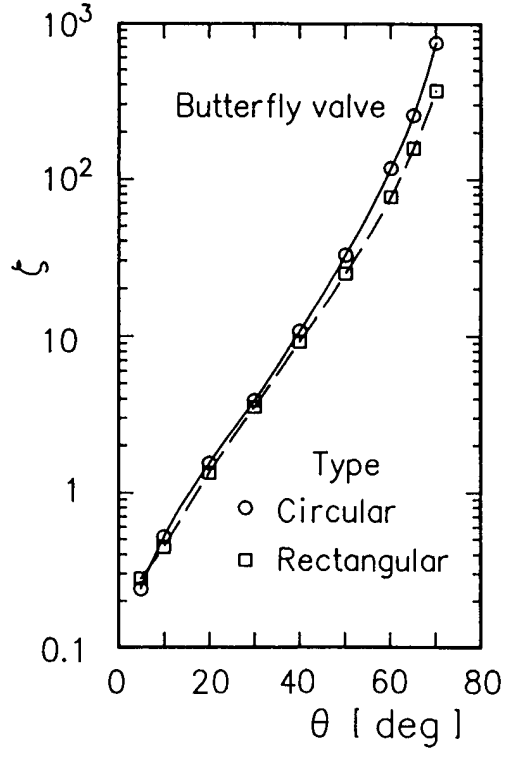

(b) Butterfly valve

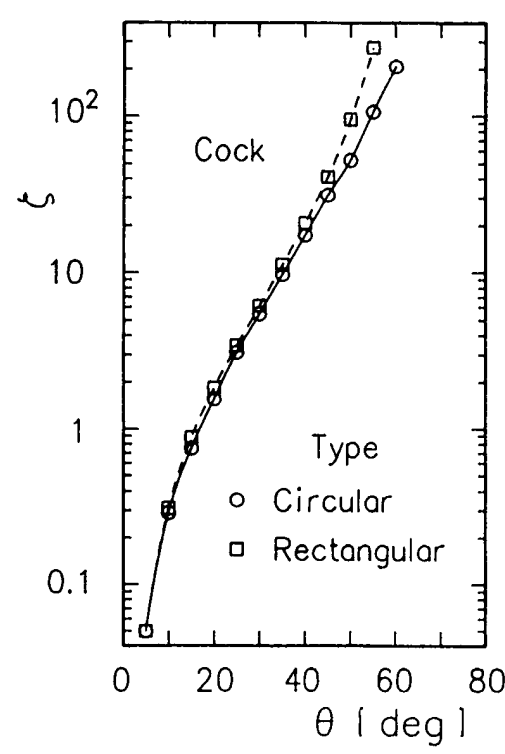

(c) Cock

Fig. 7 Hydraulic losses of pipeline devices 


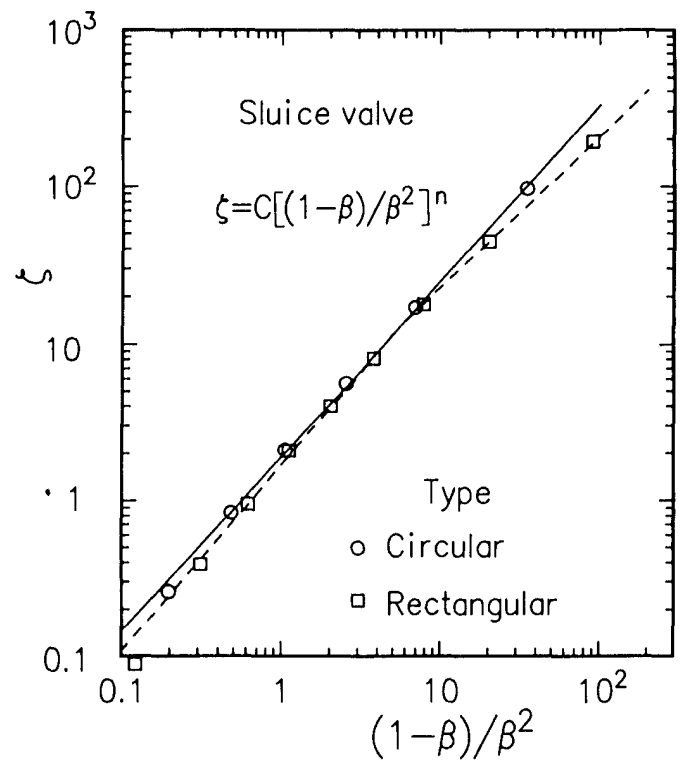

Fig. 8 Formulation of the flow resistance coefficients of sluice valve

formulation of the flow resistance coefficients of flow control devices in pipes, in the same way as that of the flow resistances in the section 3. Namely, the data of the flow resistance coefficients are arranged by a dominant factor $(1-\beta) / \beta^{2}$.

\section{2 Sluice valve}

The flow resistance coefficients of the two types of sluice valves are shown in Fig. 8. The data of the circular cross-section valve lie approximately on a straight line regardless of the opening ratio $\beta$. For the rectangular cross-section valve, the data lie on two straight lines : one is for $(1-\beta) / \beta^{2} \leqq 7.8$, the other is for $(1-\beta) / \beta^{2}>7.8$. That is to say, the slopes of the straight lines change at $\beta=0.3$. The flow resistance coefficients of the two types of sluice valves are expressed as follows.

circular cross-section :

$$
\zeta=1.9\left[(1-\beta) / \beta^{2}\right]^{1.12},(0.156 \leqq \beta \leqq 0.86),
$$

rectangular cross-section :

$$
\begin{aligned}
& \zeta=1.7\left[(1-\beta) / \beta^{2}\right]^{1.2},(0.3 \leqq \beta \leqq 0.8), \\
& \zeta=2.5\left[(1-\beta) / \beta^{2}\right]^{0.96},(0.1 \leqq \beta \leqq 0.3) .
\end{aligned}
$$

\section{3 Butterfly valve}

As shown in Fig. 9, the flow resistance coefficients of the two types of butterfly valves are given by

circular cross-section :

$$
\zeta=2.0(1-\beta) / \beta^{2},(0.134 \leqq \beta \leqq 0.913),
$$

rectangular cross-section :

$$
\zeta=1.7(1-\beta) / \beta^{2},(0.06 \leqq \beta \leqq 0.826) .
$$

The two equations are of the same form as Eq. (3) for a circular and a trapezoidal cylinder. The values of $C$ in Eq. (3) are 2.0 and 1.7, respectively.

\section{4 Cock}

The flow resistance coefficients of a cock are

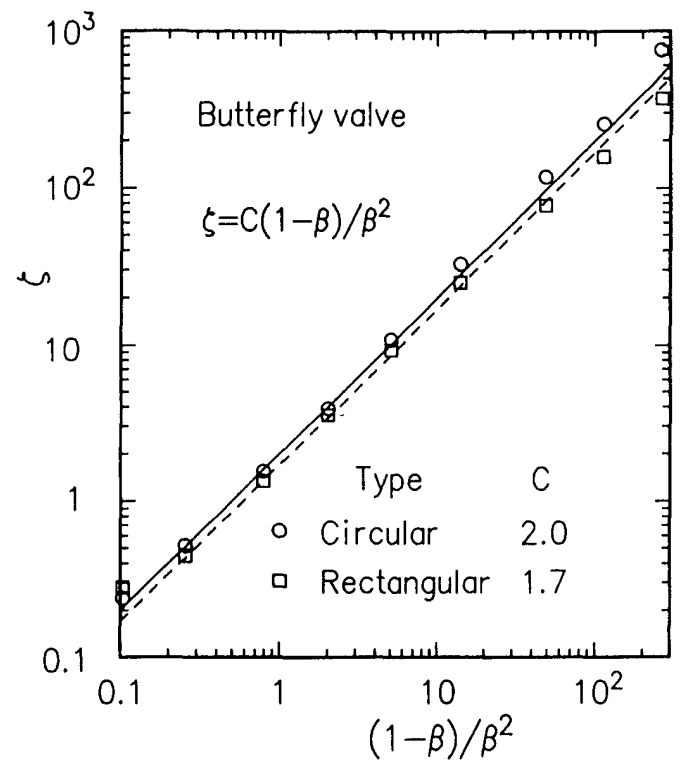

Fig. 9 Formulation of the flow resistance coefficients of butterfly valve

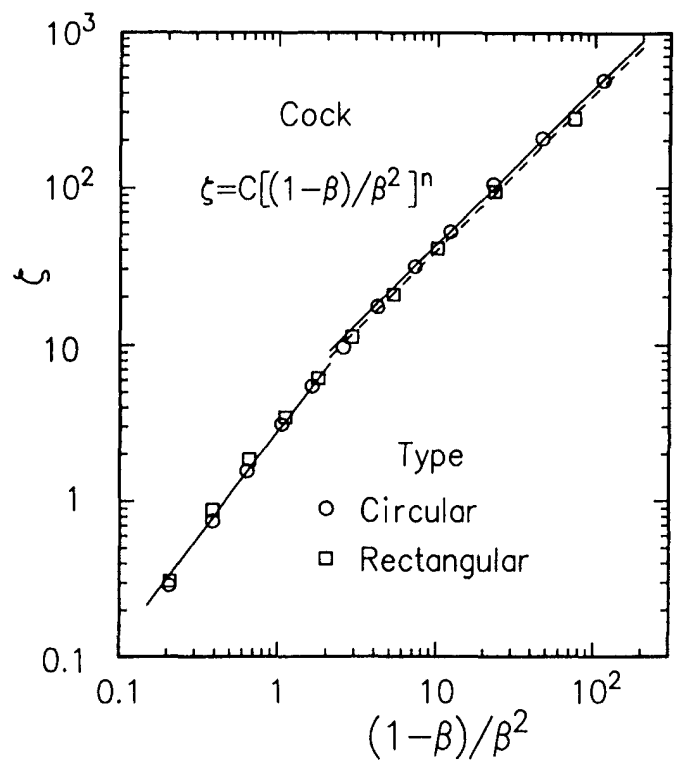

Fig. 10 Formulation of the flow resistance coefficients of cock

shown in Fig. 10. There is no significant difference in $\zeta$ between a circular-type and a rectangular-type cock. In Fig. 10, the slopes of the two lines change at about $(1-\beta) / \beta^{2}=2.0$, that is, $\beta=0.5$. At this time, the angle of cock opening $\theta$ ranges from 30 to $35^{\circ}$. The flow resistance coefficients of the two types of cocks are given by

circular cross-section:

$$
\begin{aligned}
& \zeta=2.8\left[(1-\beta) / \beta^{2}\right]^{1.4}, \quad(0.5 \leqq \beta \leqq 0.85), \\
& \zeta=4.4(1-\beta) / \beta^{2},(0.09 \leqq \beta<0.5),
\end{aligned}
$$

rectangular cross-section :

$$
\begin{aligned}
& \zeta=2.8\left[(1-\beta) / \beta^{2}\right]^{1.4},(0.5 \leqq \beta \leqq 0.85), \\
& \zeta=4.0(1-\beta) / \beta^{2},(0.11 \leqq \beta<0.5) .
\end{aligned}
$$




\section{Conclusions}

The coefficient of flow resistance of a vortex shedder in a turbulent circular pipe, $K$, was investigated in a wide range of opening ratio, and the hydraulic losses of flow control devices in a pipe, such as a valve and a cock, were formulated. The main results obtained were summarized as follows.

(1) The flow resistance coefficient $K$ of a twodimensional vortex shedder is given by

$$
\begin{aligned}
& K=0.8 \times C_{D}(1-\beta) / \beta^{2}, \\
& \beta=0.236-0.915, R e=1.9 \times 10^{4}-10^{5},
\end{aligned}
$$

where $C_{D}$ is the drag coefficient in a uniform flow. The equation agrees well with the experimental data within $\pm 5 \%$.

(2) For a circular cylinder, the flow resistance coefficient is reduced markedly at $\operatorname{Re}_{c}=U_{c} d / \nu \geqq 2 \times$ $10^{5}$, corresponding to the transition from laminar to turbulent flow in a uniform flow.

(3) The two-dimensional vortex shedder in a circular pipe can be applied to a throttle-type flowmeter. The hydraulic loss of a circular cylinder is about $40 \%$ that of an orifice.

(4) Hydraulic losses of flow control devices in pipes, that is, the flow resistance coefficients, can be formulated as

$$
\zeta=C\left[(1-\beta) / \beta^{2}\right]^{n} \text {. }
$$

(5) The flow resistance coefficient of an orifice is nearly equal to that of a trapezoidal cylinder, and is given by

$$
\zeta=1.9\left[(1-\beta) / \beta^{2}\right]^{1.07}, \beta=0.3-0.65 \text {. }
$$

(6) The flow resistance coefficient of sluice valve of circular-type is given by Eq. ( 9 ) and that of rectangular-types is given by Eqs. (10.a) and (10.b).

( 7 ) The flow resistance coefficients of a butterfly valve of circular-type and rectangular-type are given by Eqs. (11) and (12), respectively.

(8) The flow resistance coefficient of a cock of circular-type is given by Eqs. (13.a) and (13.b), and that of rectangular-type is given by Eqs. (14.a) and (14.b).

The authors wish to thank Mr. M. Kashiwagi, former student of The National Defense Academy and Messrs. N. Kanzawa and M. Yamaguchi, former students of Tokai University, for their assistance in the experiments.

\section{References}

(1) Igarashi, T., Inagaki, S. and Nishida, K., Flow Resistance of a Vortex Shedder in a Pipe, Trans. Jpn. Soc. Mech. Eng., (in Japanese), Vol. 58, No. 550, B(1992), p. 1655.

(2) Kawada, H., Komiya, K. and Yamasaki, H., Handbook of Flow Measurements, (in Japanese), (1979), p. 351, Nikkan Kougyo Sinbun-sha.

(3) JSME Data Book: Hydraulic Losses in Pipes and Ducts, (in Japanese), (1979), p. 64, Maruzen.

(4) Oki, I., Formulation of the Discharge and Resistance Coefficients for Pipe Orifice and Nozzles, Trans. Jpn. Soc. Mech. Eng., (in Japanese), Vol. 4, No. 14 (1938), p. 119.

(5) Weisbach, J., Lebrbuch der Ingeniur- und Maschinen-Mechanik, (1986), 1049, Friedrich Vieweg und Sohn. 